\title{
Ergodic Capacity of Multi-User MIMO Systems Using Pilot-Based Channel Estimation, Quantized Feedback and Outdated Feedback as well as User Selection
}

\author{
Fan Jin and Lajos Hanzo \\ School of ECS, University of Southampton, SO17 1BJ, United Kingdom. \\ Tel: +44-23-8059 3125, Fax: +44-23-8059 4508 \\ Email: \{fj1g10,lh\}@ecs.soton.ac.uk, http://www-mobile.ecs.soton.ac.uk
}

\begin{abstract}
We derive the ergodic channel capacity of a closedloop MIMO broadcast system under both random user selection (RUS) and semi-orthogonal user selection (SUS) principles, when considering the effects of channel estimation errors, channel state information (CSI) quantization errors and CSI feedback-delay. We intend to answer the question of "How many simultaneous users should be active in a time slot in order to achieve the maximum sum-rate in a MIMO broadcast system?". It is shown that we should refrain from supporting full-rank transmissions due to the excess inter-user interference caused by imperfect CSI at both the receiver and transmitter under RUS. By contrast, fullrank transmissions may be feasible under the SUS principle. An approximate ergodic capacity equation is also derived for RUS, and then an adaptive-rank transmission strategy is derived, which is capable of maximizing the achievable ergodic capacity.
\end{abstract}

\section{INTRODUCTION}

Compared to point-to-point multiple input multiple output (MIMO) communications, multiple user MIMO (MU MIMO) Downlink (DL) techniques are capable of achieving a multiple access capacity gain, which is proportional to the number of DL base station transmit antennas. Unfortunately, this benefit comes at a price of requiring channel state information at the transmitter (CSIT), albeit its provision may be deemed impractical in some systems.

Substantial efforts have been dedicated to the design of limited feedback aided systems, especially of FDD systems. The effects of imperfect CSI on the capacity of point-topoint MIMO channels were studied in [1] and [2]. As for the broadcast channel, the authors of [3] found that the feedback rate per mobile must be increased linearly with the signal to noise ratio (SNR) in order to achieve the maximum attainable multiplexing gain in the absence of sophisticated user scheduling. Most of these contributions were based on the block fading channel and assumed delay-free feedback. The authors of [4] considered the effects of imperfect and outdated CSI, and presented the comparison of different quantization strategies associated with a delayed feedback link. However, most of the previous contributions ignored the impact of the number of active users $M$ and assumed that exactly $N_{t}$ users are supported in each slot, which meant

The financial support of RC-UK under the India-Uk Advanced Technology Centre and of the EPSRC under the China-UK Science Bridge is gratefully acknowledged. full-rank transmissions. Hence the questions arise: Will fullrank transmissions perform optimally without perfect CSIT and CSIR? Will the situation be different, if sophisticated user scheduling schemes are used? In this paper we consider a general channel estimation and feedback model. The key findings of our study are as follows:

1) Unlike in [5], where perfect CSIR was considered, we invoke realistic pilot-assisted channel estimation and also take into account the effective capacity reduction imposed by the pilot overhead and feedback delay.

2) We derive the approximate ergodic capacity for both the single-user mode and multi-user mode under the RUS principle. The optimal number of active users $M^{\text {opt }}$ can be found from our approximate equations. With the approximation equations, an adaptive-rank transmission scheme is proposed.

3) We then also consider the semi-orthogonal user selection (SUS) scheme and present the simulation results to show the impact of active users $M$.

The rest of this paper is organized as follows. In Section II, we outline our system model, while in Section III, the achievable sum-rate is quantified, followed by our results in Section IV. Finally, we offer our conclusions in Section V.

\section{SYSTEM MODEL}

We consider a single-cell MIMO Gaussian broadcast channel supporting a total of $K$ users with the aid of $N_{t}$ DL transmit antennas at the base station (BS). A single receive antenna is used at each MS. We consider a homogeneous network, where all the users have the same average SNR, mobility, delay and the same number of feedback bits. We assume that $M\left(M \leqslant N_{t}\right)$ users are assigned to each timeslot. The MIMO broadcast channel (BC) is described by:

$$
y_{k}=\boldsymbol{h}_{k} \sum_{i=1}^{M} \boldsymbol{x}_{\boldsymbol{i}}+z_{k}, k=1, \ldots, M
$$

where $y_{k}$ is the DL signal received by user $k, \boldsymbol{h}_{k} \in \mathcal{C}^{1 \times N_{t}}$ is the channel coefficient vector having zero-mean, unit variance i.i.d complex Gaussian entries, $\boldsymbol{x}$ is the transmitted symbol vector obeying the power constraint $\mathbb{E}\left[\left|\boldsymbol{x}_{\boldsymbol{i}}\right|^{2}\right] \leqslant P$ and $z_{k}$ is the additive noise of variance $N_{0}$. We consider a stationary 
ergodic Gauss-Markov block-fading process model, where the fading is temporally correlated. This model may be given by:

$$
\boldsymbol{h}_{k}(t)=\alpha \boldsymbol{h}_{k}(t-1)+\boldsymbol{\Delta}(t)
$$

where $\alpha$ is the correlation coefficient, and $\boldsymbol{\Delta}(t)$ denotes the innovation process at the frame $t$ obeying the normal distribution $\boldsymbol{\Delta}(t) \backsim \mathcal{C N}\left(0,\left(1-\alpha^{2}\right) \boldsymbol{I}\right)$. The correlation coefficient $\alpha$ follows $\alpha=J_{0}(2 \pi F)$, where $J_{0}(\bullet)$ denotes the zero-order Bessel function and $F$ is the normalized Doppler frequency.

\section{A. Pilot-assisted Channel Estimation}

Each user estimates his/her own channel coefficient vector using a classic training process. The $L N_{t}$ shared pilots symbol ( $L$ symbols per antenna) are allocated in a time-orthogonal manner [6]. During the training process, user $k$ observes his/her channel at frame $t$ as: $\boldsymbol{r}_{k}(t)=\sqrt{L P} \boldsymbol{h}_{k}(t)+\boldsymbol{z}_{k}(t)$. Let us denote the estimate of the channel coefficient vector and the CSI estimation error by $\overline{\boldsymbol{h}}_{k}(t)$ and $\boldsymbol{e}_{k}(t)$, respectively. The channel vector of user $k$ at frame $t$ is modeled as:

$$
\boldsymbol{h}_{k}(t)=\overline{\boldsymbol{h}}_{k}(t)+\boldsymbol{e}_{k}(t) \text {. }
$$

The MMSE estimate of the channel vector given the observation $\left\{\boldsymbol{r}_{k}\left(t^{\prime}\right): t^{\prime}=0, \ldots, t\right\}$, written as:

$$
\overline{\boldsymbol{h}}_{k}(t)=\mathbb{E}\left[\overline{\boldsymbol{h}}_{k}(t) \mid \boldsymbol{r}_{k}(t-1), \cdots, \boldsymbol{r}_{k}(0)\right] .
$$

The elements of the CSI estimation error vector obey the distribution $\mathcal{C N}\left(0, \sigma_{1}^{2} \boldsymbol{I}_{N_{t}}\right)$, having a variance of :

$$
\begin{aligned}
\sigma_{1}^{2} & =\left(\frac{1-\alpha^{2}}{2 \alpha^{2}}\right) \times \\
& \left(-\left(1+\frac{1}{\beta}\right)+\sqrt{1+\left(\frac{1}{\beta}\right)^{2}+2\left(\frac{1}{\beta}\right) \frac{1+\alpha^{2}}{1-\alpha^{2}}}\right) .
\end{aligned}
$$

\section{B. Channel Direction Information Feedback}

Having estimated the CSI vector, each user quantizes the direction $\tilde{\boldsymbol{h}}(t)=\frac{\overline{\boldsymbol{h}}(t)}{\|\overline{\boldsymbol{h}}(t)\|}$ of its channel to a unit-norm vector $\hat{\boldsymbol{h}}(t)$ at frame $t$. The quantized vector is chosen from a predefined CB $\mathcal{B}=\left[C_{1} C_{2} \cdots C_{2^{B}}\right]$, where we have $C_{i} \in \mathcal{C}^{N_{t} \times 1}$ and $B$ is the number of $\mathrm{CB}$ index bits. The quantized CSI vector $\hat{\boldsymbol{h}}$ is determined by selecting the Code Word (CW) from $\mathcal{B}$ according to the minimum distance criterion, formulated as: $\hat{\boldsymbol{h}}(t)=C_{i}, i=\arg \max _{j=1 \cdots 2^{B}}\left|\tilde{\boldsymbol{h}}(t) C_{j}\right|$. We opted for a $\mathrm{CB}$ design based on random vector quantization (RVQ) here. Assuming that the angular difference between $\hat{\boldsymbol{h}}(t)$ and $\tilde{\boldsymbol{h}}(t)$ is $\theta$, the orthogonal decomposition of $\tilde{\boldsymbol{h}}(t)$ is expressed as:

$$
\tilde{\boldsymbol{h}}(t)=\cos \theta \hat{\boldsymbol{h}}(t)+\sin \theta \boldsymbol{g}(t),
$$

where $\boldsymbol{g}(t)$ is a unit-vector, which is orthogonal to the quantized channel vector $\hat{\boldsymbol{h}}(t)$ and $\sin ^{2} \theta$ represents the quantization error. The analysis provided in [3] shows that the upper and lower bound of the expected quantization error value of the RVQ CB is expressed as :

$$
\frac{N_{t}-1}{N_{t}} 2^{\frac{-B}{N_{t}-1}} \leq \mathbb{E}\left[\sin ^{2} \theta\right]=\sigma_{2}^{2} \leq 2^{\frac{-B}{N_{t}-1}} .
$$

\section{Delayed Feedback Link}

Once the users quantized their channel directions, they feed this information back to the DL transmitter over a delayed feedback link. We assume that the feedback link is affected by a delay of $d$ frames. As a result, the feedback delay imposes additional errors, written as: $\boldsymbol{h}(t)=\alpha^{d} \overline{\boldsymbol{h}}(t-d)+\boldsymbol{u}(t)$, where $\boldsymbol{u}(\mathrm{t})$ obey the normal distribution $\mathcal{C N}\left(0, \sigma_{3}^{2} \boldsymbol{I}_{N_{t}}\right)$, having a variance of: $\sigma_{3}^{2}=\alpha^{2 d} \sigma_{1}^{2}+\left(1-\alpha^{2}\right) \sum_{l=0}^{d-1} \alpha^{2 l}$.

\section{AChiEVABle RATE WITH IMPERFECT CSIT}

\section{A. SU MODE under the RUS principle}

The BS may construct the precoding vector using Transmit Matched Filter (TxMF) algorithm [6] according to the outdated and quantized channel direction. The received signal at transmission frame $t$ is written as:

$$
\begin{aligned}
y(t)= & \sqrt{P} \alpha^{d} \overline{\boldsymbol{h}}(t-d) \hat{\boldsymbol{h}}^{T}(t-d) s(t)+ \\
& {\left[z(t)+\sqrt{P} \boldsymbol{u}(t) \hat{\boldsymbol{h}}^{T}(t-d) s(t)\right] . }
\end{aligned}
$$

Considering the capacity loss imposed by the pilot overhead, the ergodic capacity of the SU MODE is expressed as:

$$
C_{S U}=\gamma \mathbb{E}\left[\log _{2}\left(1+\frac{P \alpha^{2}\left|\overline{\boldsymbol{h}}(t-d) \hat{\boldsymbol{h}}^{T}(t-d)\right|^{2}}{N_{0}+P\left|\boldsymbol{u}(t) \hat{\boldsymbol{h}}^{T}(t-d)\right|^{2}}\right)\right]
$$

where $\gamma=1-\frac{N_{t} L}{T}$ denotes the capacity loss owing to the pilot symbol overhead in each frame, and $T$ is the total number of symbols in a frame. We can then express the approximate ergodic capacity as:

$$
C_{S U}^{A P P}=\gamma \log _{2}\left(1+\frac{P \alpha^{2} N_{t}\left(1-\sigma_{1}^{2}\right)\left(1-\sigma_{2}^{2}\right)}{N_{0}+P \sigma_{3}^{2}}\right) .
$$

Proof: 1) Desired signal term: We first derive the approximation of the expectation of the desired signal term:

$$
\begin{aligned}
\mathbb{E}\left[P_{s}\right] & =\mathbb{E}\left[P \alpha^{2}\left|\overline{\boldsymbol{h}}(t-d) \hat{\boldsymbol{h}}^{T}(t-d)\right|^{2}\right] \\
& \stackrel{a}{=} \mathbb{E}\left[P \alpha^{2}\|\overline{\boldsymbol{h}}(t-d)\|^{2} \cos ^{2} \theta\right] \\
& \stackrel{b}{\geq} P \alpha^{2} \mathbb{E}\left[\|\overline{\boldsymbol{h}}(t-d)\|^{2}\right] \mathbb{E}\left[\cos ^{2} \theta\right] \\
& \stackrel{c}{=} P \alpha^{2} N_{t}\left(1-\sigma_{1}^{2}\right)\left(1-\sigma_{2}^{2}\right)
\end{aligned}
$$

The equality (a) follows from the Eq.(6), the inequality (b) holds owing to Jensen's inequality and the equality (c) follows from the Eq. (5) and Eq.(7).

2) Virtual noise term: As the Eq.(8) shown, user treats the estimation error as the additional noise. We then derive the approximation of the expection of the noise term:

$$
\mathbb{E}\left[N_{0}+P\left|\boldsymbol{u}(t) \hat{\boldsymbol{h}}^{T}(t-d)\right|^{2}\right] \stackrel{d}{=} N_{0}+P \sigma_{3}^{2}
$$

The equality (d) holds because that the elements of $u$ follows a normal distribution as $\mathrm{u} \mathcal{C N}\left(0, \sigma_{3}^{2} \boldsymbol{I}_{N_{t}}\right)$ and $\left|\hat{\boldsymbol{h}}^{T}\right|^{2}=1$.

Based on the analysis above, the approximate expression of SU MODE's ergodic capacity taking account to the rate loss 
owing to the piolet overhead is given by:

$$
\begin{aligned}
& C_{S U}=\gamma \mathbb{E}\left[\log _{2}\left(1+\frac{P \alpha^{2}\left|\overline{\boldsymbol{h}}(t-d) \hat{\boldsymbol{h}}^{T}(t-d)\right|^{2}}{N_{0}+P\left|\boldsymbol{u}(t) \hat{\boldsymbol{h}}^{T}(t-d)\right|^{2}}\right)\right] \\
& \geq \gamma\left[\log _{2}\left(1+\frac{\mathbb{E}\left(P \alpha^{2}\left|\overline{\boldsymbol{h}}(t-d) \hat{\boldsymbol{h}}^{T}(t-d)\right|^{2}\right]}{\mathbb{E}\left[N_{0}+P\left|\boldsymbol{u}(t) \hat{\boldsymbol{h}}^{T}(t-d)\right|^{2}\right]}\right)\right] \\
& \geq \gamma \log _{2}\left(1+\frac{P \alpha^{2} N_{t}\left(1-\sigma_{1}^{2}\right)\left(1-\sigma_{2}^{2}\right)}{N_{0}+P \sigma_{3}^{2}}\right) .
\end{aligned}
$$

The inequality (e) holds owing to Jensen's inequality. We approximate the quantization errors' upper bound in Eq. (7) by the expected value of the actual quantization errors. Then, this lower bound in Eq. (13) is seen as the approximate expression of ergodic capacity, formulated as Eq. (10).

\section{B. $M U$ MODE under the RUS principle}

Zero-forcing beamforming (ZFBF) scheme is used in this model. The received signal at transmission frame $t$ for user $k$ with imperfect CSIT is rewritten as:

$$
\begin{aligned}
y_{k}(t)= & \sqrt{P_{k}} \overline{\boldsymbol{h}}_{k}(t-d) \boldsymbol{w}_{k}(t) s_{k}(t)+ \\
& \sum_{j=1, j \neq k}^{M} \sqrt{P_{j}} \boldsymbol{h}_{k}(t) \boldsymbol{w}_{j}(t) s_{j}(t)+\hat{z}_{k} .
\end{aligned}
$$

where $\hat{z}_{k}=z_{k}+\sqrt{P_{k}} \boldsymbol{u}_{k}(t) \boldsymbol{w}_{k}(t) s_{k}(t)$. Then, the received SINR for user $k: \xi_{k}$ may be described as:

$\xi_{k}=\frac{P_{k} \alpha^{2 d}\left|\overline{\boldsymbol{h}}_{k}(t-d) \boldsymbol{w}_{k}(t)\right|^{2}}{N_{0}+P_{k}\left|\boldsymbol{u}_{k}(t) \boldsymbol{w}_{k}(t)\right|^{2}+\sum_{j=1, j \neq k}^{M} P_{j}\left|\boldsymbol{h}_{k}(t) \boldsymbol{w}_{j}(t)\right|^{2}}$.

Let us assume having an equal-power allocation scheme for each frame, the approximate ergodic capacity of the MU MODE may be formulated as Eq. (15).

Proof: 1) Desired signal term:

$$
\begin{aligned}
P_{s}= & \mathbb{E}\left[P_{k} \alpha^{2 d}\left|\overline{\boldsymbol{h}}_{k}(t-d) \boldsymbol{w}_{k}(t)\right|^{2}\right] \\
\stackrel{a}{=} & \frac{P}{M} \alpha^{2 d}\left(1-\sigma_{1}^{2}\right) N_{t}\left(\mathbb{E}\left[\left|\cos \theta \hat{\boldsymbol{h}}_{k}(t-d) \boldsymbol{w}_{k}(t)\right|^{2}\right]+\right. \\
& \left.\mathbb{E}\left[\left|\sin \theta \boldsymbol{g}_{k}(t-d) \boldsymbol{w}_{k}(t)\right|^{2}\right]\right) \\
\stackrel{b}{=} & \frac{P}{M} \alpha^{2 d}\left(1-\sigma_{1}^{2}\right)\left(\left(1-\sigma_{2}^{2}\right)\left(N_{t}-M+1\right)+\right. \\
& \left.N_{t} \sigma_{2}^{2} \mathbb{E}\left[\left|\boldsymbol{g}_{k}(t-d) \boldsymbol{w}_{k}(t)\right|^{2}\right]\right) \\
\stackrel{c}{=} & \frac{P}{M} \alpha^{2 d}\left(1-\sigma_{1}^{2}\right)\left(\left(1-\sigma_{2}^{2}\right)\left(N_{t}-M+1\right)+\sigma_{2}^{2}\right)
\end{aligned}
$$

The equality (a) holds since $\hat{\boldsymbol{h}}_{k}$ and $\boldsymbol{g}_{k}$ are unit and orthogonal. The equality (b) follows owing to the chi-square distribution $\chi_{2\left(N_{t}-M+1\right)}^{2}$ for the variable $N_{t}\left|\hat{\boldsymbol{h}}_{k} \boldsymbol{w}_{k}\right|^{2}$ [5]. Since $\boldsymbol{g}_{k}$ and $\boldsymbol{w}_{k}$ are independent and isotropically distributed on the $N_{t}$ dimensional hyperplane, $\left|\boldsymbol{g}_{k} \boldsymbol{w}_{k}\right|^{2}$ obeys the $\beta\left(1, N_{t}-1\right)$ distribution [7], we may get $\mathbb{E}\left[\left|\boldsymbol{g}_{k}(t-d) \boldsymbol{w}_{k}(t)\right|^{2}\right]=\frac{1}{N_{t}}$, then the equality (c) is derived.

2) Multi-user interference term:

$$
\begin{aligned}
& I=\mathbb{E}\left[\sum_{j=1, j \neq k}^{M} P_{j}\left|\boldsymbol{h}_{k}(t) \boldsymbol{w}_{j}(t)\right|^{2}\right] \\
& =\frac{P}{M}(M-1) \mathbb{E}\left[\left|\alpha^{d} \overline{\boldsymbol{h}}_{k}(t-d) \boldsymbol{w}_{j}(t)+\boldsymbol{u}_{k}(t) \boldsymbol{w}_{j}(t)\right|^{2}\right] \\
& \stackrel{e}{=} \frac{P}{M}(M-1)\left(\alpha^{2 d} \mathbb{E}\left[\left|\overline{\boldsymbol{h}}_{k}(t-d) \boldsymbol{w}_{j}(t)\right|^{2}\right]+\mathbb{E}\left[\left.\boldsymbol{u}_{k}(t) \boldsymbol{w}_{j}(t)\right|^{2}\right]\right) \\
& \stackrel{f}{=} \frac{P}{M}(M-1)\left(\alpha^{2 d} \mathbb{E}\left[\left\|\overline{\boldsymbol{h}}_{k}(t-d)\right\|^{2}\left|\tilde{\boldsymbol{h}}_{k}(t-d) \boldsymbol{w}_{j}(t)\right|^{2}\right]+\sigma_{3}^{2}\right) \\
& \stackrel{\underline{g}}{=} \frac{P}{M}(M-1)\left(\alpha^{2 d} N_{t}\left(1-\sigma_{1}^{2}\right) \mathbb{E}\left[\left|\sin \theta \boldsymbol{g}_{k}(t-d) \boldsymbol{w}_{k}(t)\right|^{2}\right]+\sigma_{3}^{2}\right) \\
& \stackrel{h}{=} \frac{P}{M}(M-1)\left(\alpha^{2 d} N_{t}\left(1-\sigma_{1}^{2}\right) \sigma_{2}^{2} \frac{1}{N_{t}-1}+\sigma_{3}^{2}\right)
\end{aligned}
$$

The equality (g) follow from the Eq.(6). Since $\overline{\boldsymbol{h}}_{k}(t-d)$ and $\boldsymbol{w}_{j}(t)$ are independent and the elements of these two vectors obey normal distribution with zero mean, the equality (e) holds. The equality (f) holds because that the elements of $\mathrm{u}$ follows a normal distribution as $\mathrm{u} \mathcal{C N}\left(0, \sigma_{3}^{2} \boldsymbol{I}_{N_{t}}\right)$ and $\left|\hat{\boldsymbol{w}}_{j}(t)\right|^{2}=1$. Similar with the equality (c), both $\boldsymbol{g}_{k}$ and $\boldsymbol{w}_{j}$ are unit-vectors on the $\left(N_{t}-1\right)$ dimensional hyperplane, which are orthogonal to $\hat{\boldsymbol{h}}_{k}$. As a result, the term $\left|\boldsymbol{g}_{k} \boldsymbol{w}_{j}\right|^{2}$ obeys the $\beta\left(1, N_{t}-2\right)$ distribution, we may get $\mathbb{E}\left[\left|\boldsymbol{g}_{k}(t-d) \boldsymbol{w}_{j}(t)\right|^{2}\right]=$ $\frac{1}{N_{t}-1}$, then the equality (h) is derived.

3) Virtual noise term: Similar with Eq. (12), the equation of expectation of virtual noise power is shown as:

$$
N=\mathbb{E}\left[N_{0}+P_{k}\left|\boldsymbol{u}_{k}(t) \boldsymbol{w}_{k}(t)\right|^{2}\right]=N_{0}+\frac{P}{M} \sigma_{3}^{2} .
$$

Based on the analysis above, the approximate expression of MU MODE's ergodic capacity is given by:

$$
\begin{aligned}
C_{M U} & =\gamma \mathbb{E}\left[\sum_{k=1}^{M} \log _{2}\left(1+\xi_{k}\right)\right] \\
& \stackrel{i}{\approx} M \gamma \log _{2}\left(1+\frac{\mathbb{E}\left[P_{s}\right]}{\mathbb{E}[I]+\mathbb{E}[N]}\right)
\end{aligned}
$$

Since meeting the convexity condition of each supported user's SINR can't be guaranteed, we involve the approximation (i). Also, we approximate the expected value of the quantization errors' upper bound in Eq. (7) by the expected value of the actual quantization errors encountered. Then, this lower bound in Eq. (20) may be considered to be the approximate expression of ergodic capacity, formulated as Eq. (15).

\section{Adaptive-rank Transmission Strategy}

Based on the analysis above, we derived the approximate equation of the ergodic capacity under the RUS, written as Eq. (16). We can select the optimal number of active users $M$ using Eq. (16), which is the $M$ value that maximizes the ergodic capacity, namely:

$$
M^{\text {opt }}=\arg \max _{1 \leq M \leq N_{t}} C^{A P P} .
$$

The BS firstly broadcasts a training sequence, and then each user estimates his/her own channel. Each user quantizes the CSI and then feeds back the code word index through 


$$
\begin{aligned}
& C_{M U}^{A P P}=M \gamma \log _{2}\left(1+\frac{P \alpha^{2 d}\left(1-\sigma_{1}^{2}\right)\left[\left(1-\sigma_{2}^{2}\right)\left(N_{t}-M\right)+1\right]}{M\left(N_{0}+P \sigma_{3}^{2}\right)+P \alpha^{2 d}\left(1-\sigma_{1}^{2}\right)(M-1) \frac{N_{t}}{N_{t}-1} \sigma_{2}^{2}}\right) . \\
& C^{A P P}=\left\{\begin{array}{cl}
\gamma \log _{2}\left(1+\frac{P \alpha^{2} N_{t}\left(1-\sigma_{1}^{2}\right)\left(1-\sigma_{2}^{2}\right)}{N_{0}+P \sigma_{3}^{2}}\right) & \text { if } M=1, \\
M \gamma \log _{2}\left(1+\frac{P \alpha^{2 d}\left(1-\sigma_{1}^{2}\right)\left[\left(1-\sigma_{2}^{2}\right)\left(N_{t}-M\right)+1\right]}{M\left(N_{0}+P \sigma_{3}^{2}\right)+P \alpha^{2 d}\left(1-\sigma_{1}^{2}\right)(M-1) \frac{N_{t}}{N_{t}-1} \sigma_{2}^{2}}\right) & \text { if } 1<M \leq N_{t} .
\end{array}\right.
\end{aligned}
$$

a delayed feedback link.Assuming that the BS knows all the system parameters, the BS calculate the ergodic capacity using Eq. (16) for all possible $M, M=1,2, \cdots, N_{t}$. Then the BS select the optimal $M$, using Eq.(21). Finally, the BS selects $M$ out of $K$ users and constructs the preprocessing matrix, and transmits the coded data symbols.

\section{Upgrading The RUS to the SUS Principle}

The SUS principle [8] allows the BS to assign the users that have high channel qualities have sufficiently different quantized directions. The channels of supported users are semi-orthogonal, and $\boldsymbol{h}_{k}$ and $\boldsymbol{w}_{k}$ are no longer independent. Hence, the chi-square distribution $\chi_{2\left(N_{t}-M+1\right)}^{2}$ assumption used in the RUS case no longer holds under the SUS principle.

According to the SUS principle, if $K$ is not large enough, there is a chance that we cannot support $M$ users under the SUS principle, with a predefined $M$. Hence a slightly modified SUS-algorithm may be proposed, where we sort the $\left|\hat{\boldsymbol{h}}_{k} \hat{\boldsymbol{h}}_{\mathcal{S}}^{T}\right|$ values in a decreasing order and then the BS may opt for discarding a certain fraction, for example the first half of the users in each iteration. This fraction may be deemed to be another design parameter in SUS. Since the theoretical formula is hard to derive under the imperfect and outdated CSIT, we will present the simulation results in the following section.

\section{Simulation Results}

In this section, we investigate the beneficial parameter regions for different values of $M$ in order to achieve the highest possible ergodic capacity with imperfect CSIT. In our simulations, we assume that $N_{t}=4$ and the duration of the transmission frame $T_{s}$ is $1 m s$ which is a piratical value in 3GPP LTE, each frame has $T=100$ symbols and the carrier frequency is $f_{c}=2 \mathrm{GHz}$. We consider a $5 \mathrm{~ms}$ delayed feedback link, which is a typical value used in 3GPP LTE.

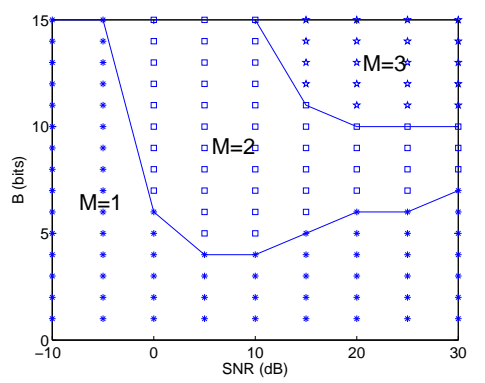

Fig. 1. The optimal number of active users $M$ at a given SNR and number of feedback bits B for achieving the highest capacity under the RUS principle, and for $L=2$ and $v=10 \mathrm{~km} / \mathrm{s}$.

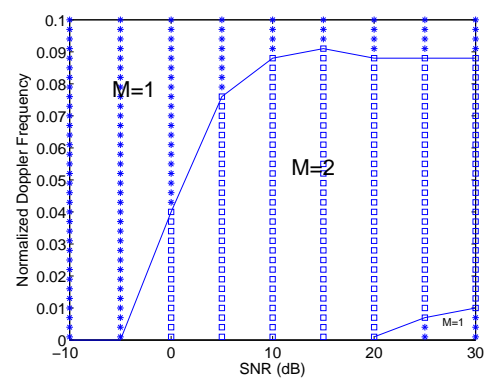

Fig. 2. The optimal number of active users $M$ at a given SNR and normalized Doppler frequencies $\mathrm{F}$ for achieving the highest capacity, for $L=2$ and $B=9$.

Fig. 1 quantifies the optimal number of active users at a given SNR and number of feedback bits B for achieving the highest capacity under $5 \mathrm{~ms}$ feedback delay. The SU MODE associated with $M=1$ has to be used in the low-SNR region below $-5 \mathrm{~dB}$ and in part of the medium-to-high SNR region where the number of feedback bits is lower than 6 bits. By contrast, predominantly $M=2$ has to be used in the mediumSNR region between $0 \mathrm{~dB}$ and $30 \mathrm{~dB}$, when the number of quantization bits is higher than $B=4$ bits and lower than $B=11$. High rank transmissions $(M \geq 3)$ are not beneficial until the SNR becomes sufficiently high and simultaneously, the number of quantization bits is sufficiently high, namely for $S N R>15 d B, B>10$. Full-rank transmissions $(M=4)$ were never found to be beneficial in our investigations for the parameters considered.

Fig. 2 shows the associated parameter regions for different normalized Doppler frequencies under $5 \mathrm{~ms}$ feedback delays. Higher $F$ implies that the channel coefficient vector fluctuates more rapidly. Hence, the correlation coefficient $r$ will be reduced. Note the SU MODE will benefit in the $S N R \leq$ $-5 d B$ region and $M=2$ will result in an increased capacity at $S N R \geq 0 d B$ region, when we set $B=9$ and $F \leq 0.04$. When $F$ becomes bigger, the SU MODE has to be used even when $S N R \geq 10 d B$ when $F \geq 0.085$.

Let us verify the approximate ergodic capacity results of Section III in Fig. 3. Observe that the approximate formula of Eq. (16) is quite accurate in the low-to-medium SNR range, say for $-10 \mathrm{~dB}$ to $15 \mathrm{~dB}$. By contrast, there is a gap between the approximate capacity and the simulation results for SNRs in the region of $15 \mathrm{~dB}$ to $30 \mathrm{~dB}$ for the MU MODE, where the approximate capacity becomes a lower bound. This is because the approximation introduced in Eq.(7) and Eq. (20).

Fig. 4 compares the ergodic capacity for different transmission strategies. In the low-SNR region, i.e. for $S N R<$ $0 d B$, adaptive-rank transmissions may have the same ergodic capacity as SU transmission. The ergodic capacity of adaptive- 


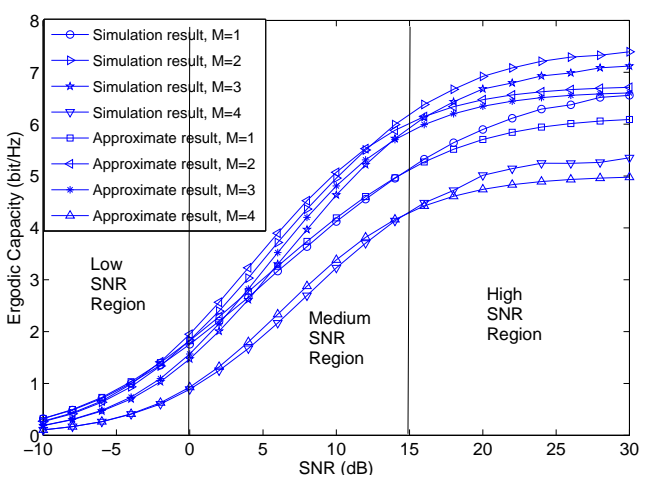

Fig. 3. Simulation results and approximate ergodic capacity vs. SNR results under the RUS for different number of active users $M$, for $L=2, B=9$, $v=10 \mathrm{~km} / \mathrm{s}$ and $d=5 \mathrm{~ms}$.

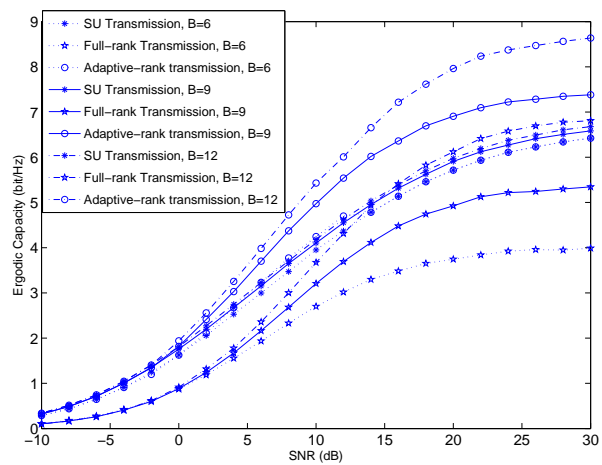

Fig. 4. The ergodic capacity vs. SNR performance using adaptive-rank transmissions, compared to the SU transmission and full-rank transmission, where $L=2, v=10 \mathrm{~km} / \mathrm{s}$ and $d=5 \mathrm{~ms}$ are developed.

rank transmission is expected to be higher than that of SU transmission and that of full-rank transmissions in the medium-high SNR region.

Unlike the discussion above, we now demonstrate the beneficial parameter regions under the SUS principle. Fig. 5 characterizes the regions of different $M$ values for different number of feedback bits $B$ and the total number of supported users $K$. For a small value of $K=100$, the SU MODE has to be used for $S N R \leq-5 d B$. Supporting $M=3$ active users will lead to the highest ergodic capacity for most parameter values owing to the associated multi-user diversity gain. Unlike for RUS, full-rank transmissions ( $M=4)$ may be activated, when the number of quantization bits is sufficiently high $(B \geq 12)$ in the SNR region of $\rho \geq 20 d B$. Also, for a given $B$, the SU MODE based region is gradually reduced, as $K$ increases. By contrast, the high-rank transmission region $(M \geq 3)$ is expanded upon increasing.

\section{CONCLUSION}

In this treatise, the achievable ergodic capacity of multiuser MIMO systems was derived under both the RUS and the modified SUS regime, when considering the effects of channel estimation errors, quantization errors, feedback delay and the capacity loss due to the pilot overhead. Based on the approximate expressions of Eq. (16), as well as the

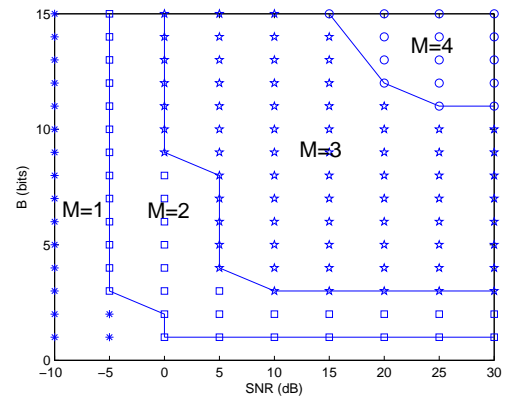

(a) Different $B, L=2, K=100$ and $d=5$

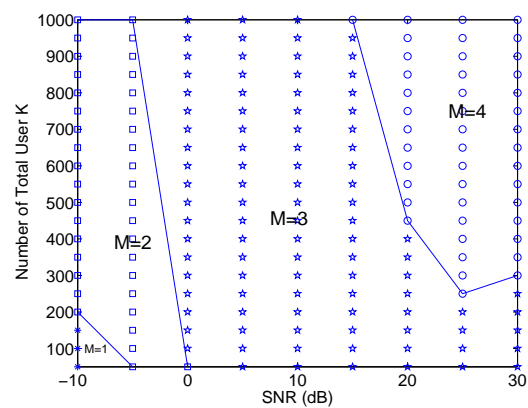

(b) Different $K, L=10, N b=9$ and $d=5$

Fig. 5. The beneficial regions of supporting different number of active users $M$ with a realistically estimated channel, channel quantization and feedback delay under the modified SUS principle

simulation results, we concluded that full-rank transmission do not achieve the highest rates. However, for sufficiently high values of $K$ and $B$, full-rank transmissions combined with our SUS regime may become beneficial. Also, the proposed adaptive-rank transmission strategy is capable of achieving higher ergodic capacity comparing to the SU transmission and full-rank transmission.

\section{REFERENCES}

[1] B. Hassibi and B. M. Hochwald, "How Much Training is Needed in Multiple-Antenna Wireless Links?," IEEE Transactions on Information Theory, vol. 49, pp. 951-963, Apr. 2003.

[2] M. Medard, "The Effect Upon Channel Capacity in Wireless Communications of Perfect and Imperfect Knowledge of the Channel," IEEE Transactions on Information Theory, vol. 46, pp. 933-946, May 2000.

[3] N. Jindal, "MIMO Broadcast Channels With Finite-Rate Feedback," IEEE Transactions on Information Theory, vol. 52, pp. 5045-5060, Nov. 2006.

[4] G. Caire, N. Jindal, and M. Kobayashi, "Multiuser MIMO Achievable Rates with Downlink Training and Channel State Feedback," IEEE Transactions on Information Theory, vol. 56, pp. 2845-2866, Jun. 2010.

[5] J. Zhang, M. Kountouris, J. G. Andrews, and R. W. Heath, "Achievable Throughput of Multi-Mode Multiuser MIMO With Imperfect CSI Constraints," in Proc. IEEE International Symposium on Information Theory, pp. 2659-2663, July 2009.

[6] L. Hanzo, M. Munster, B. J. Choi, and T. Keller, OFDM and MC-CDMA for Broadcasting Multi-User Communications, WLANs and Broadcasting. John Wiley-IEEE Press, 2003.

[7] T. Yoo, N. Jindal, and A. Goldsmith, "Multi-Antenna Downlink Channels with Limited Feedback and User Selection," IEEE Journal on Selected Areas in Communications, vol. 25, pp. 1478-1491, Sept. 2007.

[8] T. Yoo and A. Goldsmith, "Sum-rate Optimal Multi-Antenna Downlink Beamforming Strategy based on Clique Search," in Proc. IEEE Global Telecommunications Conference, vol. 3, p. 5, Nov. 2005. 